\title{
Efficacy and Tolerance of Interferon $\beta$ Plus Ribavirin Treatment for Chronic Hepatitis C Patients with Depression or Thrombocytopenia Comparison with Pegylated Interferon $\alpha$ Plus Ribavirin Treatment
}

Hiroaki Ikezaki, Norihiro Furusyo*, Eiichi Ogawa, Motohiro Shimizu, Satoshi Hiramine, Kazuya Ura, Fujiko Mitsumoto, Kouji Takayama, Kazuhiro Toyoda, Masayuki Murata and Jun Hayashi

Department of General Internal Medicine, Kyushu University Hospital, Fukuoka, Japan

\begin{abstract}
Objective: Limited data has been reported comparing natural human interferon $\beta$ (nIFN $\beta$ ) and pegylated IFN- $\alpha$ (PEG-IFNa) when Ribavirin (RBV) is combined. This case-control study was done to compare the efficacy and adverse effects of a combination treatment of nIFN $\beta$ or PEG-IFNa plus RBV for chronic hepatitis $C$ patients.

Methods: Sixty patients with chronic hepatitis C, 42 infected with hepatitis C virus (HCV) genotype 1 and 18 infected with genotype 2 , were treated with nIFN $\beta$ plus RBV. Of them, $23(38.3 \%)$ suffered pre-treatment severe depression. Their data was compared with 60 undepressed patients treated with a combination of PEG-IFNa plus RBV. nIFN $\beta$ was given intravenously and PEG-IFNa was injected subcutaneously.

Results: Sustained virological response (undetectable HCV RNA at 24 weeks after the end of treatment) did not significantly differ between the nIFN $\beta$ and PEG-IFNa treated patients (genotype $1,21.4 \%$ vs. $33.3 \%, P=0.328$; genotype $2,72.2 \%$ vs. $88.9 \%$, respectively, $P=0.402$ ). None of the $n I F N \beta$ treated patients showed exacerbation of depression, while $7(11.7 \%)$ of 60 PEG-IFNa treated patients developed severe depression or malaise. The platelet count of nIFN $\beta$ treated patients increased to higher than baseline after week 8 , but the platelet count of PEG-IFNa treated patients decreased throughout the treatment. There were significant differences of the changes of platelet counts between the both groups throughout the treatment (all $P<0.001$ ).
\end{abstract}

Conclusion: $\mathrm{nIFN} \beta$ plus RBV treatment was well tolerated by chronic hepatitis C patients with depression or thrombpcytopenia.

Keywords: Interferon $\beta$; Ribavirin; Chronic hepatitis C; Depression; Thrombocytopenia

\section{Introduction}

Hepatitis $\mathrm{C}$ virus (HCV) infection is a major cause of chronic hepatitis, cirrhosis, and hepatocellular carcinoma (HCC) and thus represents a significant public health problem [1,2]. The primary objective of treatment for chronic hepatitis $\mathrm{C}$ is to eradicate HCV, achieve Sustained Virological Response (SVR), and to prevent progression to cirrhosis or HCC [3]. We previously reported that the eradication of HCV infection decreases the occurrence of hepatocellular carcinoma [4].

Pegylated interferon a (PEG-IFNa) plus Ribavirin (RBV) combination treatment has improved the SVR rate for patients with chronic hepatitis C [5]. Previous studies reported that $25-40 \%$ of such patients discontinued the combination treatment due to adverse effects, such as psychological problems or cytopenia [5-8]. Patients with depression are not considered suitable for this combination treatment. In Japan, natural human interferon $\beta$ (nIFN $\beta$ ) and RBV combination treatment has been approved and recommended for depressed patients with chronic hepatitis $C$, and some studies have shown that nIFN $\beta$ plus RBV treatment has equivalent efficacy and mild adverse effects [9-11].

This case-control study was done to compare the efficacy and safety of nIFN $\beta$ plus RBV combination treatment for Japanese chronic hepatitis $\mathrm{C}$ patients with that of PEG-IFNa plus RBV combination treatment.

\section{Patients and Methods}

\section{Patients}

A total of 60 Japanese patients with chronic hepatitis C treated with nIFN $\beta$ plus RBV between 2009 and 2012 at Kyusyu University Hospital were enrolled in this study. Of the 60 patients, 42 infected with HCV genotype $1 \mathrm{~b}$ were placed in group $\mathrm{B} 1$ and 18 with genotype 2 in group B2. To compare the clinical efficacy and safety of the treatment, we retrospectively selected 60 patients treated with PEG-IFNa2b and RBV treatment, matched with the group B for genotype, sex, age, and body weight before treatment (Group A1: HCV genotype $1 \mathrm{~b}, \mathrm{n}=42$ and A2: HCV genotype 2, $n=18$ ). Patients who had severe depression with suicidal ideation and / or attempt were excluded.

This study was carried out in accordance with the principles of the Declaration of Helsinki as revised in 2000 and was approved by the Kyushu University Hospital Ethics Committee. Informed consent was obtained from all patients before treatment.

Treatment protocol, dose reduction, and discontinuation of treatment

Treatment periods were 24 for genotype 2 and 48 weeks for

*Corresponding author: Norihiro Furusyo, Department of General Internal Medicine, Kyushu University Hospital, 3-1-1 Maidashi, Higashi-ku, Fukuoka, 812-8582, Japan, Tel: +81-92-642-5909; Fax: +81-92-642-5916; E-mail: furusyo@gim.med.kyushu-u.ac.jp

Received April 03, 2014; Accepted April 22, 2014; Published May 05, 2014

Citation: Ikezaki H, Furusyo N, Ogawa E, Shimizu M, Hiramine S, et al. (2014) Efficacy and Tolerance of Interferon $\beta$ Plus Ribavirin Treatment for Chronic Hepatitis C Patients with Depression or Thrombocytopenia Comparison with Pegylated Interferon a Plus Ribavirin Treatment. J Liver 3: 155. doi:10.4172/21670889.1000155

Copyright: ( 2014 lkezaki H, et al. This is an open-access article distributed under the terms of the Creative Commons Attribution License, which permits unrestricted use, distribution, and reproduction in any medium, provided the original author and source are credited. 
Citation: Ikezaki H, Furusyo N, Ogawa E, Shimizu M, Hiramine S, et al. (2014) Efficacy and Tolerance of Interferon $\beta$ Plus Ribavirin Treatment for Chronic Hepatitis C Patients with Depression or Thrombocytopenia Comparison with Pegylated Interferon $\alpha$ Plus Ribavirin Treatment. J Liver 3: 155. doi:10.4172/2167-0889.1000155

Page 2 of 7

genotype 1 patients, respectively, with a subsequent 24 -week follow-up period. Two types of interferon were prescribed, as below.

Groups B1 and B2: nIFN $\beta$ (Feron; Toray Industries Inc., Tokyo, Japan) was given intravenously at a dose of 6 million units daily for first 4 weeks of treatment, followed by three times a week for the remaining 20-44 weeks.

Groups A1 and A2: PEG-IFNa2b (Peg-Intron; MSD Co., Tokyo, Japan) was injected subcutaneously at a dose of $1.5 \mu \mathrm{g} / \mathrm{kg}$ once a week for 24-48 weeks.

RBV (Rebetol; MSD Co., Tokyo, Japan) was given orally twice a day at a total dose of 600-1,000 mg for 24-48 weeks. The initial dose was adjusted according to body weight $(600 \mathrm{mg}$ for patients weighing $\leq 60$ $\mathrm{kg}, 800 \mathrm{mg}$ for those between 60 and $80 \mathrm{~kg}$, and $1,000 \mathrm{mg}$ for those $\geq 80$ $\mathrm{kg}$ ). Both nIFN $\beta$ or PEG-IFNa2b and RBV were concurrently initiated. The above durations and dosages are those approved by the Japanese Ministry of Health, Labor, and Welfare [12].

The dose of nIFN $\beta$ or PEG-IFNa2b was reduced if a patient had an adverse psychological effect, the white blood cell count fell below 1500 $\times 10^{6} / \mathrm{L}$, or the platelet count fell below $50 \times 10^{9} / \mathrm{L}$. Likewise, the dose of RBV was reduced if the hemoglobin level decreased to under 100 $\mathrm{g} / \mathrm{L}$. Treatment was discontinued if the hemoglobin level, white blood cell count, or platelet count fell below $85 \mathrm{~g} / \mathrm{L}, 1000 \times 10^{6} / \mathrm{L}$, or $25 \times 10^{9}$ $/ \mathrm{L}$, respectively.

\section{HCV RNA detection and clinical evaluation}

Blood samples were collected from the patients just before treatment, at weeks $4,8,12,24$, and 48 of the treatment, at the end of treatment (EOT), and at 24 weeks after EOT. The serum HCV RNA level at each point was determined by quantitative real time Polymerase Chain Reaction (PCR) assay (COBAS TaqMan HCV assay; Roche Diagnostics) with a linear dynamic range of 1.2 to $7.8 \log _{10} \mathrm{IU} / \mathrm{mL}$ [13]. Biochemical and hematological tests were performed once each month during treatment. All were measured by standard laboratory techniques in our hospital laboratory. Liver biopsy was performed for $103(85.8 \%)$ of the 120 patients by experienced hepatologists and the stage of fibrosis (F0-4) and the grade of activity (A0-3) were established according to the METAVIR score [14].

\section{Interleukin $28 \mathrm{~B}$ and inosine triphosphate pyrophosphatase}

Human genomic DNA was extracted from peripheral blood. Genotyping by the Single-Nucleotide Polymorphism (SNP) of the interleukin 28B (IL28B) (rs8099917) and inosine triphosphate pyrophosphatase (ITPA) (rs1127354) genes was done using the TaqMan Allelic Discrimination Demonstration Kit (7500 Real-Time PCR System; Applied Biosystems, Foster City, CA). Patients were genotyped as IL28B TT, TG, or GG and as ITPA CC, CA, or AA at the polymorphic site [15].

\section{Virological response}

SVR was defined as undetectable HCV RNA at 24 weeks after the end of treatment. Early virological response during treatment was categorized as follows: Rapid Virological Response (RVR), undetectable HCV RNA at week 4. Complete early viological response (cEVR), detectable HCV RNA at week 4 but undetectable at week 12. Relapse was defined as undetectable HCV RNA at the end of treatment, but non-SVR. Non-response was defined as detectable HCV RNA through treatment. Non-SVR was defined as not achieving SVR, including relapse and non-response.

\section{Assessment of depression}

A questionnaire survey was conducted when physicians perceived the necessity or patients complained depressive symptom, using the Beck Depression Inventory II (BDI-II) and the Pittsburgh Sleep Quality Index (PSQI) $[16,17]$. Patients with a BDI-II score of 14 or more were considered to have the onset of depression symptoms. Patients with a PSQI score of 11 or more were identified as having sleep disorder.

\section{Statistical analysis}

All statistical analyses were performed using JMP ver. 9 (SAS Institute Inc., Cary, NC). Data are reported as mean \pm standard error (SE), median [first quartile, third quartile], or percentage for each category. Spearman correlation coefficient analysis was used to analyze patient characteristics. The student's $t$ test and the Mann-Whitney $U$ test were used to compare between-group differences. A $P$ value of $<0.05$ was considered statistically significant.

\section{Results}

\section{Clinical characteristics}

Table 1 shows the pretreatment clinical characteristics of the patients of groups B and A. No significant differences in baseline serum HCV RNA levels, histology of fibrosis, or distribution of IL28B or ITPA genotype were found between the $\mathrm{B} 1$ and $\mathrm{A} 1$ or $\mathrm{B} 2$ and $\mathrm{A} 2$ groups. Group B1 had a significantly higher rate of history of IFN therapy, higher levels of aspartate aminotransferase and $\alpha$-fetoprotein, and a significantly lower platelet count than group A1. Group B2 had a significantly higher rate of depression and a lower rate of IL28B TT genotype than group A2. The discontinuation rates of group B1 and B2 during prior IFN treatment were higher than those of group A1 and A2, but there was no significant difference.

\section{Efficacy of treatment}

Tables 2 and 3 show the virological response to both treatments. There was no significant difference in the SVR rates of groups B1 and $\mathrm{A} 1(21.4 \%$ vs. $33.3 \%, P=0.328)$ or groups $\mathrm{B} 2$ and $\mathrm{A} 2(72.2 \%$ vs. $88.9 \%, P=0.402)$. Patients with RVR achieved SVR significantly more often than patients without RVR in group B1 and B2 $(P=0.001$ and 0.029 , respectively), but no significance was found for groupA1 and A2 $(P=0.106$ and 0.065 , respectively). Patients with cEVR achieved SVR significantly more often than patients without cEVR in group $\mathrm{B} 1, \mathrm{~B} 2$ and $\mathrm{A} 1 \quad(P<0.001$ and $P=0.008$ and 0.020 , respectively $)$, but no significance was found for group A2 $(P=0.111)$. In B1, IL28B TT patients showed significantly higher SVR rates than IL28B nonTT patients $(33.3 \%, 8$ of 24 patients and $0.0 \%, 0$ of 14 , respectively, $P=0.017)$. In group B2 and A1, IL28B TT patients showed higher SVR rates than $I L 28 B$ non-TT patients (B2; 75.0\%, 9 of 12 vs. $60.0 \%, 3$ of 5, and $A 1 ; 34.6 \%, 9$ of 26 vs. $25.0 \%, 2$ of 8 , respectively) but the difference was not significant $(P=0.600$ and $P>0.999$, respectively). In group A2, all patients determined $I L 28 B$ genotype had IL28B TT genotype.

\section{Safety and tolerance of nIFN $\beta$ plus RBV treatment}

There was no significant difference in the discontinuation rates between group $\mathrm{B} 1$ and $\mathrm{A} 1(P=0.164)$ or group $\mathrm{B} 2$ and $\mathrm{A} 2(P=0.486)$. Of the 42 group B1 patients, 11 (26.2\%) discontinued the treatment because of malaise ( 3 patients at weeks $14,20,24)$, hyperthyroidism (1 patient at week 9), poor response (4 patients at weeks 15, 16, 19, 38 ), new occurrence of HCC (2 patients at weeks 3, 25), and for personal reasons (1 patient at week 24). Of the 42 group A1 patients, $5(11.9 \%)$ discontinued treatment, two because of malaise at week 38 
Citation: Ikezaki H, Furusyo N, Ogawa E, Shimizu M, Hiramine S, et al. (2014) Efficacy and Tolerance of Interferon $\beta$ Plus Ribavirin Treatment for Chronic Hepatitis C Patients with Depression or Thrombocytopenia Comparison with Pegylated Interferon a Plus Ribavirin Treatment. J Liver 3: 155. doi:10.4172/2167-0889.1000155

Page 3 of 7

\begin{tabular}{|c|c|c|c|c|c|c|}
\hline Groups & Group B1 & Group A1 & & Group B2 & Group A2 & \\
\hline Treatment & $\mathrm{nlFN} \beta+\mathrm{RBV}$ & PEG-IFNa2b + RBV & $P$ value & $\mathrm{nIFN} \beta+\mathrm{RBV}$ & PEG-IFNa2b + RBV & $P$ value \\
\hline Studied $\mathrm{n}$ & 42 & 42 & matched & 18 & 18 & matched \\
\hline Depression, $\mathrm{n}(\%)$ & $12(28.6)$ & $5(11.9)$ & 0.102 & $11(61.1)$ & $1(0.6)$ & $<0.001$ \\
\hline HCV genotype & \multicolumn{2}{|c|}{$1 \mathrm{~b}$} & & \multicolumn{2}{|c|}{2} & \\
\hline Men, n (\%) & $16(38.1)$ & $16(38.1)$ & matched & $7(38.9)$ & $7(38.9)$ & matched \\
\hline Prior IFN treatment history, $\mathrm{n}(\%)$ & $34(81.0)$ & $14(33.3)$ & $<0.001$ & $10(55.6)$ & $7(38.9)$ & 0.505 \\
\hline $\begin{array}{l}\text { Prior treatment outcome } \\
\text { (Relapse / Non response / Discontinuation) }\end{array}$ & $5 / 22 / 7$ & $4 / 8 / 2$ & 0.518 & $3 / 3 / 4$ & $2 / 5 / 0$ & 0.116 \\
\hline Prior treatment discontinuation, $\mathrm{n}(\%)$ & $7(20.6)$ & $2(14.3)$ & 0.611 & $4(40.0)$ & $0(0.0)$ & 0.056 \\
\hline Age (years) & $66[60,71]$ & $65[59,71]$ & 0.648 & $60[50,69]$ & $57[48,65]$ & 0.537 \\
\hline Body weight (kg) & $59.2[50.8,69.3]$ & $57.2[51.4,68.1]$ & 0.858 & $54.1[46.9,67.8]$ & $56.4[49.2,67.0]$ & 0.764 \\
\hline Body mass index $\left(\mathrm{kg} / \mathrm{m}^{2}\right)$ & $23.1[21.4,26.4]$ & $23.4[21.3,25.6]$ & 0.906 & $22.9[19.7,25.6]$ & $23.3[20.9,26.4]$ & 0.448 \\
\hline \multicolumn{7}{|l|}{ Histology } \\
\hline Activity (A0/A1/A2/A3) & $1 / 17 / 16 / 1$ & $0 / 13 / 20 / 3$ & 0.549 & $0 / 6 / 8 / 1$ & $0 / 4 / 11 / 2$ & 0.531 \\
\hline Fibrosis (F0/F1/F2/F3/F4) & $1 / 16 / 8 / 8 / 2$ & $2 / 18 / 7 / 4 / 5$ & 0.667 & $2 / 5 / 5 / 0 / 3$ & $5 / 7 / 1 / 0 / 4$ & 0.246 \\
\hline Not determined histology study & 7 & 6 & & 3 & 1 & \\
\hline IL28B (TT / TG·GG) & $24 / 14$ & $26 / 8$ & 0.218 & $12 / 5$ & $15 / 0$ & 0.042 \\
\hline ITPA (CC / CA·AA) & $22 / 16$ & $21 / 13$ & 0.626 & $14 / 3$ & $11 / 4$ & 0.472 \\
\hline Not determined IL28B or ITPA phenotype & 4 & 8 & & 1 & 1 & \\
\hline Serum HCV RNA level (log IU/mL) & $6.25[5.68,6.83]$ & $6.05[5.54,6.50]$ & 0.175 & $5.99[4.85,6.76]$ & $6.23[5.20,6.60]$ & 0.812 \\
\hline Serum albumin $(\mathrm{g} / \mathrm{L})$ & $39[37,41]$ & $40[37,44]$ & 0.063 & $42[39,45]$ & $43[39,44]$ & 0.691 \\
\hline Aspartate aminotransferase (IU/L) & $67[48,100]$ & $49[38,78]$ & 0.022 & $41[27,91]$ & $56[36,71]$ & 0.874 \\
\hline Alanine aminotransferase (IU/L) & $66[38,94]$ & $54[35,94]$ & 0.607 & $40[23,129]$ & $52[31,100]$ & 0.788 \\
\hline Y-glutamyl transpeptidase (IU/L) & $55[34,98]$ & $41[26,67]$ & 0.171 & $40[21,69]$ & $35[18,84]$ & 0.937 \\
\hline eGFR $\left(\mathrm{mL} / \mathrm{min} / 1.73 \mathrm{~m}^{2}\right)$ & $78.7[69.0,88.5]$ & $72.0[61.5,87.5]$ & 0.168 & $81.7[69.5,91.7]$ & $83.2[75.8,91.4]$ & 0.420 \\
\hline White blood cell $\left(\times 10^{6} / \mathrm{L}\right)$ & $4325[3798,5300]$ & $4610[3838,5850]$ & 0.431 & $4870[3500,6020]$ & $5000[3750,6800]$ & 0.448 \\
\hline Hemoglobin $(\mathrm{g} / \mathrm{L})$ & $135 \pm 2$ & $134 \pm 2$ & 0.872 & $135 \pm 4$ & $131 \pm 4$ & 0.438 \\
\hline Platelet $(\times 10 \% / L)$ & $110[84,161]$ & $169[143,214]$ & $<0.001$ & $166[111,202]$ & $167[128,196]$ & 0.681 \\
\hline$\alpha$-fetoprotein $(\mathrm{ng} / \mathrm{mL})$ & $10.5[5.1,29.1]$ & $6.5[3.6,13.3]$ & 0.069 & $4.8[3.0,26.6]$ & $4.9[2.5,12.7]$ & 0.764 \\
\hline Initiation dose of RBV / body weight $(\mathrm{mg} / \mathrm{kg}$ ) & $12.1 \pm 0.2$ & $11.2 \pm 0.2$ & 0.069 & $12.2 \pm 0.3$ & $11.4 \pm 0.3$ & 0.730 \\
\hline
\end{tabular}

IFN, interferon; nIFN $\beta$, natural interferon $\beta$; PEG-IFNa2b, pegylated interferon $\alpha 2 \mathrm{~b}$; RBV, ribavirin; $\mathrm{HCV}$, hepatitis $\mathrm{C}$ virus; IL28B, interleukin 28B; ITPA, inosine triphosphate pyrophosphatase; eGFR, estimated glomerular filtration rate

Liver activity histology was classified as : A1, mild ; A2, moderate ; A3 severe.

Liver fibrosis histology was classified as : F1, periportal expansion ; F2, portoportal septa ; F3, portocentral linkage or bridging fibrosis ; F4, cirrhosis.

Data are shown as median [first-quartile, third-quartile], mean \pm standard error, and number.

$\mathrm{P}$ values were calculated between Group B1 and A1 or between Group B2 and A2.

Table 1: Comparison of baseline clinical characteristics of patients with nIFN $\beta$ plus RBV and PEG-IFNa2b plus RBV.

\begin{tabular}{|c|c|c|c|c|c|c|}
\hline & \multicolumn{2}{|c|}{$\begin{array}{c}\text { Group B1 (nIFN } 3+\text { RBV }) \\
(n=42)\end{array}$} & \multirow[b]{2}{*}{$P$ value } & \multicolumn{2}{|c|}{$\begin{array}{l}\text { Group A1 (PEG-IFNa2b + RBV) } \\
(n=42)\end{array}$} & \multirow[b]{2}{*}{$P$ value } \\
\hline & $\begin{array}{l}\text { SVR } \\
(n=9)\end{array}$ & $\begin{array}{c}\text { non-SVR } \\
(\mathrm{n}=33)\end{array}$ & & $\begin{array}{c}\text { SVR } \\
(n=14)\end{array}$ & $\begin{array}{c}\text { non-SVR } \\
(\mathrm{n}=28)\end{array}$ & \\
\hline Pretreatment depression, $\mathrm{n}(\%)$ & $2(22.2)$ & $10(30.3)$ & $>0.999$ & $1(7.1)$ & $4(14.3)$ & $>0.999$ \\
\hline Exacerbation or new developed depression, $\mathrm{n}(\%)$ & $0(0.0)$ & $0(0.0)$ & $>0.999$ & $1(7.1)$ & $6(21.4)$ & 0.242 \\
\hline Men, $\mathrm{n}(\%)$ & $2(22.2)$ & $14(42.4)$ & 0.442 & $6(42.9)$ & $10(35.7)$ & 0.742 \\
\hline Age (years) & $64[57,71]$ & $68[60,72]$ & 0.539 & $63[58,67]$ & $67[60,71]$ & 0.186 \\
\hline Age over $65, \mathrm{n}(\%)$ & $4(44.4)$ & $22(66.7)$ & 0.265 & $5(35.7)$ & $17(60.7)$ & 0.192 \\
\hline Body mass index $\left(\mathrm{kg} / \mathrm{m}^{2}\right)$ & $26.4[22.7,28.1]$ & $22.9[20.9,25.4]$ & 0.138 & $23.1[21.1,24.8]$ & $23.4[21.4,27.2]$ & 0.386 \\
\hline RVR / cEVR, n & $5 / 3$ & $1 / 2$ & $<0.001$ & $2 / 9$ & $0 / 10$ & 0.011 \\
\hline IL28B (TT / TG·GG) & $8 / 0$ & $16 / 14$ & 0.017 & $9 / 2$ & $17 / 6$ & $>0.999$ \\
\hline ITPA (CC / CA·AA) & $5 / 3$ & $17 / 13$ & $>0.999$ & $7 / 4$ & $14 / 9$ & $>0.999$ \\
\hline $\begin{array}{l}\text { Histology Fibrosis } \\
\text { (F0 / F1 / F2 / F3 / F4) }\end{array}$ & $0 / 3 / 2 / 2 / 0$ & $1 / 13 / 6 / 6 / 2$ & 0.907 & $1 / 7 / 2 / 2 / 1$ & $1 / 11 / 5 / 2 / 4$ & 0.862 \\
\hline Prior interferon history, $\mathrm{n}(\%)$ & $5(55.6)$ & $29(87.9)$ & 0.050 & $2(14.3)$ & $12(42.9)$ & 0.089 \\
\hline $\begin{array}{l}\text { Prior treatment outcome } \\
\text { (Relapse / Non response) }\end{array}$ & $3 / 2$ & $2 / 27$ & 0.015 & $2 / 0$ & $2 / 10$ & 0.066 \\
\hline $\begin{array}{l}\text { Serum HCV RNA level } \\
\text { (log IU/mL) }\end{array}$ & $5.12 \pm 0.47$ & $6.42 \pm 0.10$ & 0.006 & $6.02 \pm 0.21$ & $6.10 \pm 0.11$ & 0.521 \\
\hline Serum albumin $(g / L)$ & $40 \pm 2$ & $38 \pm 1$ & 0.324 & $41 \pm 1$ & $40 \pm 1$ & 0.698 \\
\hline Aspartate aminotransferase (IU/L) & $58[27,76]$ & $76[54,102]$ & 0.064 & $47[38,76]$ & $49[37,79]$ & 0.968 \\
\hline Alanine aminotransferase (IU/L) & $42[25,83]$ & $69[42,118]$ & 0.086 & $52[34,98]$ & $54[36,92]$ & 0.894 \\
\hline Y-glutamyl transpeptidase (IU/L) & $48[15,80]$ & $56[36,110]$ & 0.191 & $30[23,63]$ & $48[26,88]$ & 0.173 \\
\hline
\end{tabular}


Citation: Ikezaki H, Furusyo N, Ogawa E, Shimizu M, Hiramine S, et al. (2014) Efficacy and Tolerance of Interferon $\beta$ Plus Ribavirin Treatment for Chronic Hepatitis C Patients with Depression or Thrombocytopenia Comparison with Pegylated Interferon a Plus Ribavirin Treatment. J Liver 3: 155. doi:10.4172/2167-0889.1000155

Page 4 of 7

\begin{tabular}{|c|c|c|c|c|c|c|}
\hline eGFR (mL/min/1.73m²) & $77.8[68.6,82.5]$ & $78.7[69.0,89.7]$ & 0.709 & $77.4[66.3,87.7]$ & $68.8[60.8,90.7]$ & 0.584 \\
\hline White blood cell $(/ \mu \mathrm{L})$ & $4100[3690,5180]$ & $4360[3830,5300]$ & 0.510 & $4610[3840,5510]$ & $4620[3830,5950]$ & 0.979 \\
\hline Hemoglobin (g/L) & $132 \pm 5$ & $136 \pm 3$ & 0.480 & $137 \pm 3$ & $132 \pm 3$ & 0.603 \\
\hline Platelet $\left(\times 10^{4} / \mu \mathrm{L}\right)$ & $14.4[8.5,18.2]$ & $10.7[8.4,15.2]$ & 0.416 & $20.0[16.6,21.5]$ & $15.0[13.4,17.8]$ & 0.023 \\
\hline a-fetoprotein (ng/mL) & $5.7[3.2,8.7]$ & $12.9[6.3,42.5]$ & 0.011 & $5.6[3.1,7.4]$ & $6.9[3.7,15.4]$ & 0.225 \\
\hline Adherence rates of IFN (\%) & 100 & 100 & $>0.999$ & $98.7 \pm 2.7$ & $87.8 \pm 3.2$ & 0.034 \\
\hline Initiation dose of RBV / body weight $(\mathrm{mg} / \mathrm{kg}$ ) & $12.1 \pm 0.4$ & $12.1 \pm 0.3$ & 0.915 & $11.9 \pm 0.5$ & $10.9 \pm 0.2$ & 0.093 \\
\hline Adherence rates of RBV (\%) & $100[73.7,103.4]$ & $100[94.2,100]$ & 0.919 & $64.7[49.4,100]$ & $41.6[26.7,77.8]$ & 0.049 \\
\hline
\end{tabular}

Data are shown as median [first-quartile, third-quartile], mean \pm standard error, and number.

IFN, interferon; HCV, hepatitis C virus; SVR, sustained virological response; RVR, rapid virological response; cEVR, complete early virological response; IL28B, interleukin 28B; ITPA, inosine triphosphate pyrophosphatase; nIFN $\beta$, natural interferon $\beta$; PEG-IFNa2b, pegylated interferon $\alpha 2 \mathrm{~b}$; RBV, ribavirin; eGFR, estimated glomerular filtration rate

Liver fibrosis histology was classified as F1, periportal expansion ; F2, portoportal septa ; F3, portocentral linkage or bridging fibrosis ; F4, cirrhosis.

$P$ values were calculated between SVR and non-SVR patients of each groups.

Table 2: Results of patients infected HCV genotype 1 treated with nIFN $\beta$ plus RBV and PEG-IFNa2b plus RBV.

\begin{tabular}{|c|c|c|c|c|c|c|}
\hline & \multicolumn{2}{|c|}{$\begin{array}{l}\text { Group B2 (nIFN } \beta+\text { RBV) } \\
(n=18)\end{array}$} & \multirow[b]{2}{*}{$P$ value } & \multicolumn{2}{|c|}{$\begin{array}{l}\text { Group A2 (PEG-IFNa2b + RBV) } \\
(n=18)\end{array}$} & \multirow[b]{2}{*}{$P$ value } \\
\hline & $\begin{array}{l}\text { SVR } \\
(n=13)\end{array}$ & $\begin{array}{l}\text { non-SVR } \\
(n=5)\end{array}$ & & $\begin{array}{l}\text { SVR } \\
(n=16)\end{array}$ & $\begin{array}{l}\text { non-SVR } \\
(n=2)\end{array}$ & \\
\hline Pretreatment depression, n (\%) & $8(61.5)$ & $3(60.0)$ & $>0.999$ & $1(6.3)$ & $0(0.0)$ & $>0.999$ \\
\hline $\begin{array}{l}\text { Exacerbation or newly developed depression, } \\
\mathrm{n}(\%)\end{array}$ & $0(0.0)$ & $0(0.0)$ & $>0.999$ & $0(0.0)$ & $0(0.0)$ & $>0.999$ \\
\hline Men, $\mathrm{n}(\%)$ & $5(38.5)$ & $2(6.1)$ & $>0.999$ & $7(43.8)$ & $0(0.0)$ & 0.497 \\
\hline Age (years) & $56.0 \pm 4.2$ & $60.8 \pm 4.6$ & 0.882 & $54.4 \pm 3.1$ & $61.0 \pm 6.0$ & 0.439 \\
\hline Age over $65, \mathrm{n}(\%)$ & $4(30.8)$ & $2(40.0)$ & $>0.999$ & $4(25.0)$ & $1(50.0)$ & 0.490 \\
\hline Body mass index $(\mathrm{kg} / \mathrm{m} 2)$ & $22.6 \pm 1.1$ & $24.4 \pm 1.7$ & 0.430 & $23.8 \pm 0.7$ & $22.9 \pm 2.7$ & 0.527 \\
\hline RVR / cEVR, n & $9 / 3$ & $0 / 1$ & 0.006 & $13 / 3$ & $0 / 1$ & 0.006 \\
\hline IL28B (TT / TG·GG) & $9 / 3$ & $3 / 2$ & 0.600 & $13 / 0$ & $2 / 0$ & \\
\hline ITPA (CC / CA·AA) & $11 / 1$ & $3 / 2$ & 0.191 & $9 / 4$ & $2 / 0$ & $>0.999$ \\
\hline Histology fibrosis (F0 / F1 / F2 / F3 / F4) & $1 / 5 / 4 / 0 / 2 / 1$ & $1 / 0 / 1 / 0 / 1 / 2$ & 0.439 & $5 / 7 / 1 / 0 / 2 / 0$ & $0 / 0 / 0 / 0 / 2 / 0$ & 0.061 \\
\hline Prior IFN treatment history, $\mathrm{n}(\%)$ & $7(53.8)$ & $3(60.0)$ & $>0.999$ & $7(43.8)$ & $0(0.0)$ & 0.497 \\
\hline $\begin{array}{l}\text { Prior treatment outrcome } \\
\text { (Relapse / Non response) }\end{array}$ & $2 / 5$ & $1 / 2$ & $>0.999$ & $2 / 5$ & $0 / 0$ & $>0.999$ \\
\hline $\begin{array}{l}\text { Serum HCV RNA level } \\
(\log I U / m L)\end{array}$ & $5.08 \pm 0.50$ & $6.43 \pm 0.26$ & 0.126 & $5.86 \pm 0.27$ & $5.84 \pm 0.66$ & 0.622 \\
\hline Serum albumin $(\mathrm{g} / \mathrm{L})$ & $42[41,46]$ & $42[34,48]$ & 0.843 & $43[40,45]$ & $32[29,34]$ & 0.032 \\
\hline Aspartate aminotransferase (IU/L) & $40[26,84]$ & $72[29,113]$ & 0.622 & $51[33,67]$ & $75[62,87]$ & 0.206 \\
\hline Alanine aminotransferase (IU/L) & $41[30,135]$ & $32[18,119]$ & 0.554 & $52[27,101]$ & $52[33,70]$ & 0.725 \\
\hline Y-glutamyl transpeptidase (IU/L) & $35[18,49]$ & $74[44,185]$ & 0.038 & $46[22,95]$ & $15[13,17]$ & 0.079 \\
\hline eGFR (mL/min/1.73m2) & $82.7 \pm 3.8$ & $80.3 \pm 7.7$ & 0.657 & $87.0 \pm 4.5$ & $78.7 \pm 3.7$ & 0.440 \\
\hline White blood cell $(/ \mu \mathrm{L})$ & $5072 \pm 467$ & $3968 \pm 428$ & 0.168 & $5197 \pm 436$ & $4895 \pm 1905$ & 0.888 \\
\hline Hemoglobin (g/L) & $135 \pm 4$ & $135 \pm 7$ & 0.921 & $133 \pm 4$ & $114 \pm 4$ & 0.079 \\
\hline Platelets $(\times 104 / \mu \mathrm{L})$ & $17.2 \pm 1.6$ & $12.6 \pm 2.2$ & 0.168 & $17.8 \pm 1.4$ & $10.9 \pm 0.2$ & 0.106 \\
\hline a-fetoprotein (ng/mL) & $3.5[2.5,15.7]$ & $7.4[4.0,34.3]$ & 0.168 & $4.0[2.4,7.4]$ & $13.9[13.6,14.1]$ & 0.058 \\
\hline Adherence rates of IFN (\%) & 100 & 100 & $>0.999$ & $96.7 \pm 3.5$ & $96.1 \pm 0.3$ & $>0.999$ \\
\hline Initiation dose of RBV / body weight (mg/kg) & $12.4 \pm 0.3$ & $11.6 \pm 0.5$ & 0.182 & $11.5 \pm 0.4$ & $10.8 \pm 0.0$ & 0.527 \\
\hline Adherence rates of RBV (\%) & $100[93.8,100]$ & $100[88.0,100]$ & $>0.999$ & $75.7[57.8,99.0]$ & $31.8[13.6,50.0]$ & 0.078 \\
\hline
\end{tabular}

HCV, hepatitis C virus ; SVR, sustained virological response ; RVR, rapid virological response ; cEVR, complete early virological response ; IL28B, interleukin $28 \mathrm{~B}$; ITPA inosine triphosphate pyrophosphatase ; IFN, interferon ; nIFN $\beta$, natural interferon $\beta$; PEG-IFN, pegylated interferon $\alpha 2 b$; RBV, ribavirin ; eGFR, estimated glomerular filtration rate

Liver fibrosis histology was classified as : F1, periportal expansion ; F2, portoportal septa ; F3, portocentral linkage or bridging fibrosis ; F4, cirrhosis

Data are shown as median [first-quartile, third-quartile], mean \pm standard error, and number.

$P$ values were calculated between SVR and non-SVR patients of each groups.

Table 3: Results of patients infected HCV genotype 2 treated with nIFN $\beta$ plus RBV and PEG-IFNa2b plus RBV.

and three because of poor response at weeks 16,18 , and 24 . Of the 18 group B2 patients, 2 (11.1\%) discontinued treatment, one because of poor response at week 28 and the other because of the recurrence of maxillary cancer at week 29 week. Of the 18 group A2 patients, none discontinued treatment. Although the total rate of discontinuation of the 60 group B patients $(21.6 \%)$ was significantly higher than that of the 60 group A patients $(8.3 \%)(P=0.041)$, there was no significant difference in the rates of discontinuation because of treatment-related side effect (malaise and hypothyroidism) between groups $B(n=4,6.7 \%)$ and $\mathrm{A}(\mathrm{n}=2,3.3 \%)(P=0.402)$.

Of the 60 group B patients, none had exacerbated or newly developed depression during treatment, but $7(11.7 \%)$ of the 60 group A patients developed depression during treatment $(P=0.002)$ and all of 7 required to reduce the dose of PEG-IFNa2b.

Of the 60 group $B$, none required a reduction of the dose of nIFN $\beta$. 
Citation: Ikezaki H, Furusyo N, Ogawa E, Shimizu M, Hiramine S, et al. (2014) Efficacy and Tolerance of Interferon $\beta$ Plus Ribavirin Treatment for Chronic Hepatitis C Patients with Depression or Thrombocytopenia Comparison with Pegylated Interferon a Plus Ribavirin Treatment. J Liver 3: 155. doi:10.4172/2167-0889.1000155

Page 5 of 7

However, in the 42 group A1 patients, the adherence rate to PEGIFNa2b was $85.2[72.2,96.9] \%$ (median [first quartile, third quartile]), and in the 18 group A2 patients, the rate was $96.0[87.8,108.5] \%$. There was a significant difference in the adherence rates to IFN between groups $\mathrm{B} 1$ and $\mathrm{A} 1(P=0.005)$, but not between groups $\mathrm{B} 2$ and $\mathrm{A} 2$ $(P=0.230)$. In 60 group A patients, 14 required a reduction of the dose of PEG-IFNa2b, 7 required because they developed depression, and other 7 required because their platelet counts fell below $50 \times 10^{9} / \mathrm{L}$. The SVR rate of these 14 group A, PEG-IFNa2b reduction patients was significantly lower (2 patients, $14.3 \%$ ) than that of 46 group A patients who did not required a reduction (28 patients, $60.9 \%)(P=0.002)$. None required discontinuation the therapy because of adverse effect.

There was a significant difference in the adherence rates to RBV between groups B1 and A1 $(100[86.8,100] \%$ and $31.9[21.2,50.3] \%$, respectively $(P<0.001))$ and between groups B2 and A2 (100 [96.9, $100] \%$ and $50.0[27.7,55.2] \%$, respectively $(P=0.002))$.

\section{Influence of nIFN $\beta$ to blood count}

The platelet count of group B increased to higher than baseline after week 8 , but the platelet count of group A decreased throughout the treatment. Significant differences were found between groups B and A throughout the treatment (all $P<0.001$ ). Figure 1 shows the differences in the on-treatment changes of platelet count by ITPA genotype. The platelet counts of ITPA non-CC patients were lower than those of ITPA CC patients (significantly at weeks 4,12 and $24, P=0.013,0.102,0.039$ and 0.008 at weeks $4,8,12$ and 24, respectively) for group B (Figure 1a). The ITPA non-CC patients had a significantly higher decrease than the ITPA CC patients at week $4(P=0.001)$, but there were no significant differences after week $8(P=0.141,0.329$ and 0.281 at weeks 8,12 and 24 , respectively) for group A (Figure $1 \mathrm{~b}$ ).

There was no significant difference in the decrease of hemoglobin level at weeks 4,8 or 12 between groups B and A. At week 24, the decrease of the hemoglobin level of group A became significantly higher than that of group $\mathrm{B}(P=0.048)$, even though the adherence rate to RBV of group A was significantly lower than group B. Figure 2 shows on-treatment differences in the decrease of hemoglobin levels, by ITPA genotype. The hemoglobin levels of ITPA CC patients decreased significantly more than those of ITPA non-CC patients $(P<0.001$ at week 4 and $P=0.002,0.005$, and 0.022 at weeks 8,12 and 24 , respectively) in group B (Figure $2 \mathrm{a}$ ). The hemoglobin of ITPA CC patients decreased significantly more than those of ITPA non-CC patients at week $4(P<0.001)$, but there was no significant difference between the genotypes after week $8(P=0.252,0.621$ and 0.787 at weeks 8,12 and 24, respectively) in group A (Figure $2 b$ ).

\section{Discussion}

The four major findings of the present study are as follows. First, the efficacy of the nIFN $\beta$ plus RBV treatment was equivalent to that of PEG-IFNa2b plus RBV treatment. Second, none of the patients treated with nIFN $\beta$ plus RBV had exacerbated or newly developed depression. Third, the platelet count of patients treated with nIFN $\beta$ plus RBV increased to higher than baseline after week 8 whereas they fell with PEG-IFN2b plus RBV. Finally, significantly fewer ITPA nonCC patients than ITPA CC patients treated with nIFN $\beta$ plus RBV had a decrease in the hemoglobin level.

Although the overall discontinuation rates for nIFN $\beta$ plus RBV treatment were slightly higher than for PEG-IFNa2b and RBV treatment, the number of patients discontinued because of interferoninduced side effects, such as malaise or thyroid disease, was equivalent. And the platelet count of patients taking nIFN $\beta$ increased to higher than baseline. The SVR rates for nIFN $\beta$ plus RBV treatment and PEGIFNa2b plus RBV treatment were equivalent for chronic hepatitis $\mathrm{C}$ patients, however, because the mean age of the patients with HCV genotype 1 was over 65 years, the SVR rate of these patients was low as the previous studies $[8,18]$. These results indicate that nIFN $\beta$ plus RBV combination treatment is effective and safe for patients with chronic hepatitis $\mathrm{C}$, in particular for those with depression or thrombocytopenia. (a) Group B: nIFN $\beta+$ RBV

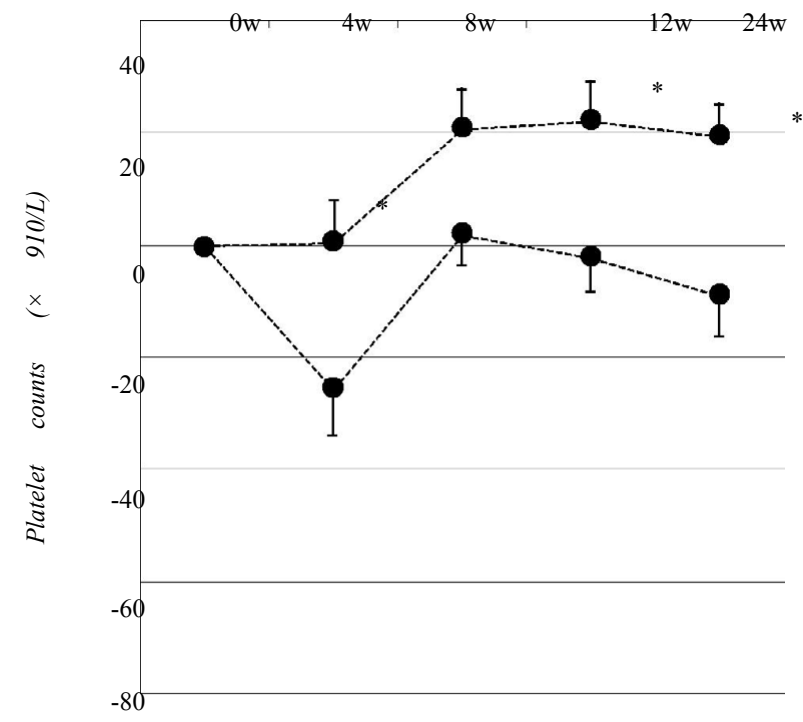

(b) Group A: PEG-IFN $\alpha 2 b+R B V$

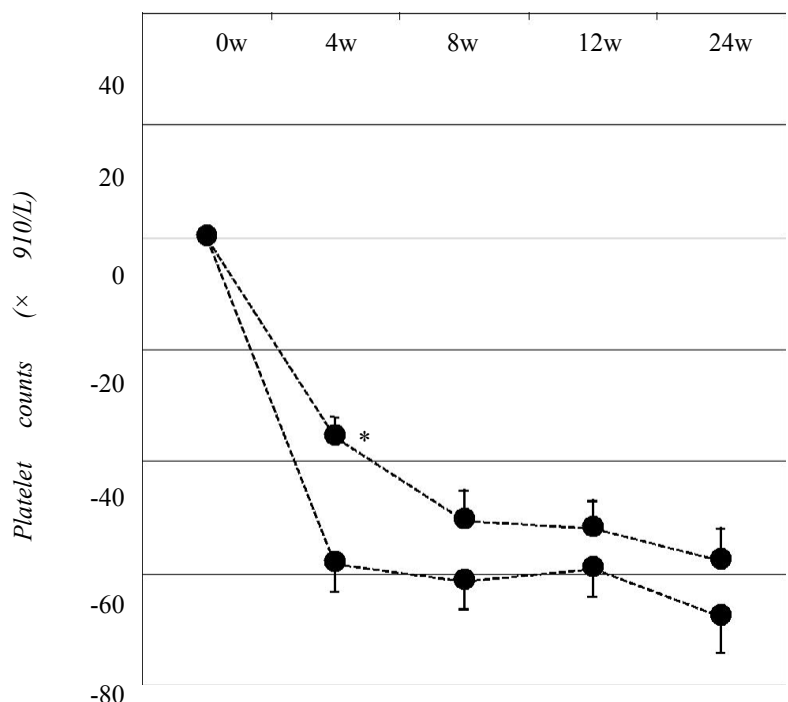

$-80$ 
Citation: Ikezaki H, Furusyo N, Ogawa E, Shimizu M, Hiramine S, et al. (2014) Efficacy and Tolerance of Interferon $\beta$ Plus Ribavirin Treatment for Chronic Hepatitis C Patients with Depression or Thrombocytopenia Comparison with Pegylated Interferon $\alpha$ Plus Ribavirin Treatment. J Liver 3: 155. doi:10.4172/2167-0889.1000155

Page 6 of 7

(a) Group B: nIFN $\beta+$ RBV

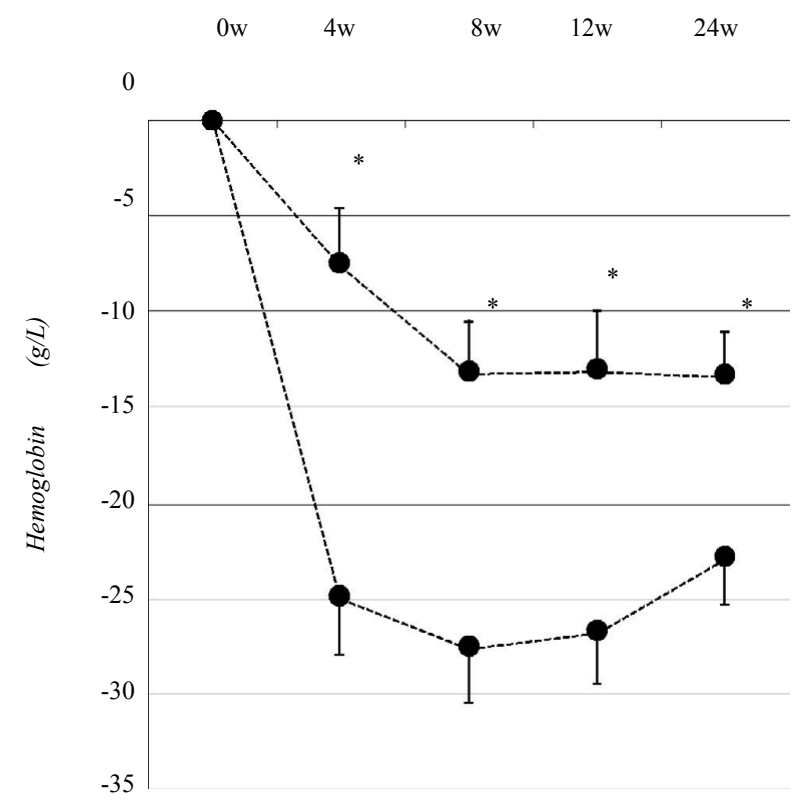

(b) Group A: PEG-IFN $\alpha 2 b+$ RBV

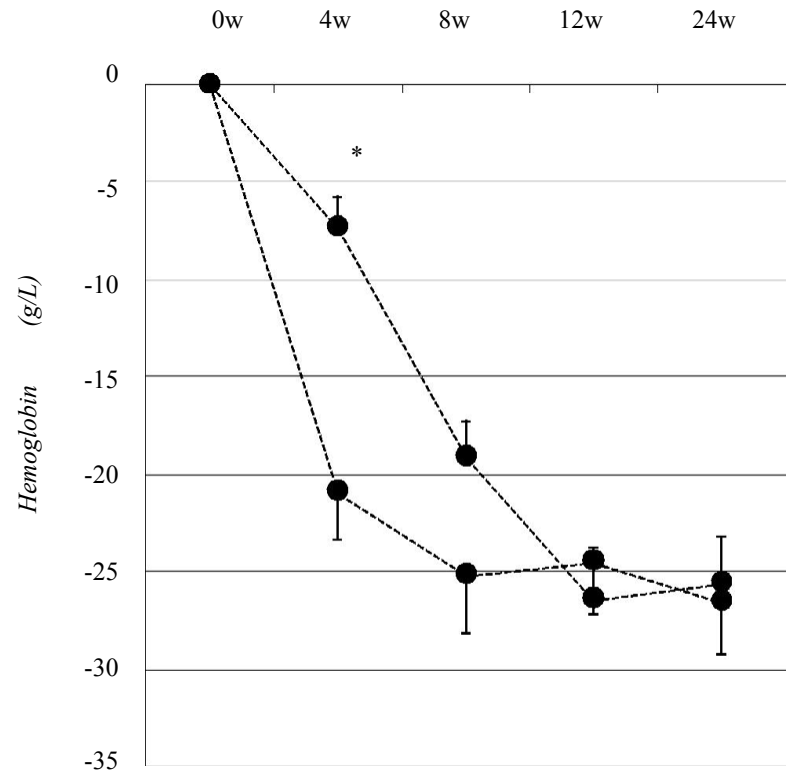

------ ITPA CC ----- ITPA nonCC

Figure 2: Decreases of the hemoglobin level by ITPA genotype.

PEG-IFNa is known to often cause and exacerbate psychological problems (the range was reported to be from 30 to $80 \%$ ) $[19,20]$. The incidence of nIFN $\beta$-induced psychological problems has been reported to be from 0 to $10 \%$ [9-11,21]. In this study, $38.3 \%$ (23 of 60$)$ of the patients who received nIFN $\beta$ plus RBV treatment suffered depression before treatment, but all of them completed treatment without increasing antidepressants or reduction of nIFN $\beta$. Of the patients who received PEG-IFNa2b and RBV treatment, 11.6\% (7 of 60) developed depression during treatment. The difference in the frequency of nIFN $\beta$ induced and PEG-IFNa-induced psychological problems may be related to the higher elevation of serum level of interleukin-1 receptor antagonist (IL-1Ra) with anti-inflammatory effects by nIFN $\beta$ than by PEG-IFNa. The ratio of interleukin-1 $\beta$ to IL-1Ra is maintained within normal range because IL-1Ra does not decrease when nIFN $\beta$ is used $[22,23]$.

PEG-IFNa often causes cytopenia. This side effects often lead to decrease the treatment dosage. In this study, patients who underwent nIFN $\beta$ plus RBV treatment had an increased platelet count after week 8 of treatment. Moreover, the platelet count of patients with ITPA CC was higher than at baseline after week 4 of treatment. In contrast, the platelet count of patients who underwent treatment with PEGIFNa2b and RBV decreased throughout treatment, regardless of ITPA genotype. Fewer patients who underwent nIFN $\beta$ plus RBV treatment had a decrease in hemoglobin level than did patients who underwent PEG-IFNa2b and RBV, even though the adherence rate to RBV was significantly higher for the patients treated with nIFN $\beta$ plus RBV than for those treated with PEG-IFNa2b and RBV. Although the mechanism of the platelet count increase of the patients who receive nIFN $\beta$ plus RBV treatment remains to be clarified, the physiological linkage of hematopoietic factors may explain this phenomonon. Thrombosis has been reported in patients with iron deficiency anemia and Bilic and Bilic reported that the amino acid sequence homology of thrombopoietin and erythropoietin might explain it [24-26]. From our results, the ITPA CC might be associated with the increase of platelet count during nIFN $\beta$ plus RBV treatment. Further studies will be necessary to clarify the relation between nIFN $\beta$ plus RBV treatment and changes of platelet count.

The histories of prior IFN treatment of patients of both groups were different. However, patients of groups B were considered to be less tolerant to IFN treatment than those of groups A, because the discontinuation rates of prior IFN treatment among groups B were higher than those among groups A. Thus, we did not consider that that difference might weaken our result that nIFN $\beta$ plus ribavirin treatment had enough tolerance.

Because patients must go to a hospital for treatment three times a week, nIFN $\beta$ is somewhat more inconvenient than PEG-IFNa. However, this inconvenience is offset by the milder and fewer nIFN $\beta$ related side effects and the increased platelet count during treatment.

This study has some limitations. One is the small number of patients. Although the SVR rates were not statistically different between groups B and A, there is a probability of difference when the number of patients were more. Further study with more patients will be necessary. Another is that the IL28B genotype was not determined for some patients, so careful interpretation must be made of the evaluation of the influence of $I L 28 B$ on the efficacy of both treatments. Further study to determine the $I L 28 B$ of all patients is desirable. The final limitation is that the study was not of a randomized design. Unfortunately, for ethical reasons it is very difficult to conduct a randomized study in Japan.

In conclusion, nIFN $\beta$ plus RBV combination treatment is an optional treatment for chronic hepatitis $\mathrm{C}$, especially for patients with depression or thrombopenia. 
Citation: Ikezaki H, Furusyo N, Ogawa E, Shimizu M, Hiramine S, et al. (2014) Efficacy and Tolerance of Interferon $\beta$ Plus Ribavirin Treatment for Chronic Hepatitis C Patients with Depression or Thrombocytopenia Comparison with Pegylated Interferon a Plus Ribavirin Treatment. J Liver 3: 155. doi:10.4172/2167-0889.1000155

\section{References}

1. Hayashi J, Furusyo N, Ariyama I, Sawayama Y, Etoh Y, et al. (2000) A relationship between the evolution of hepatitis $C$ virus variants, liver damage, and hepatocellular carcinoma in patients with hepatitis C viremia. $\mathrm{J}$ Infect Dis 181: 1523-1527.

2. Kanwal F, Hoang T, Kramer JR, Asch SM, Goetz MB, et al. (2011) Increasing prevalence of $\mathrm{HCC}$ and cirrhosis in patients with chronic hepatitis $\mathrm{C}$ virus infection. Gastroenterology 140: 1182-1188.

3. Mazzella G, Accogli E, Sottili S, Festi D, Orsini M, et al. (1996) Alpha interferon treatment may prevent hepatocellular carcinoma in HCV-related liver cirrhosis. J Hepatol 24: 141-147.

4. Ogawa E, Furusyo N, Kajiwara E, Takahashi K, Nomura H, et al. (2013) Efficacy of pegylated interferon alpha-2b and ribavirin treatment on the risk of hepatocellular carcinoma in patients with chronic hepatitis $\mathrm{C}$ : A prospective, multicenter study. J Hepatol 58: 495-501.

5. Fried MW, Shiffman ML, Reddy KR, Smith C, Marinos G, et al. (2002) Peginterferon alfa-2a plus ribavirin for chronic hepatitis $C$ virus infection. N Eng J Med 347: 975-982.

6. Manns MP, McHutchison JG, Gordon SC, Rustgi VK, Shiffman M, et al. (2001) Peginterferon alfa-2b plus ribavirin compared with interferon alfa-2b plus ribavirin for initial treatment of chronic hepatitis $\mathrm{C}$ : a randomised trial. Lance 358: 958-965.

7. Schaefer M, Schmidt F, Folwaczny C, Lorenz R, Martin G, et al. (2003) Adherence and mental side effects during hepatitis $C$ treatment with interferon alfa and ribavirin in psychiatric risk groups. Hepatology 37: 443-451.

8. Kainuma M, Furusyo N, Kajiwara E, Takahashi K, Nomura H, et al. (2010) Pegylated interferon $\mathrm{a}-2 \mathrm{~b}$ plus ribavirin for older patients with chronic hepatitis C. World J Gastroenterol 16: 4400-4409.

9. Arase Y, Suzuki F, Akuta N, Sezaki H, Suzuki Y, et al. (2010) Efficacy and safety of combination therapy of natural human interferon beta and ribavirin in chronic hepatitis $C$ patients with genotype $1 \mathrm{~b}$ and high virus load. Inter Med 49: 957-963

10. Arase Y, Suzuki F, Akuta N, Sezaki H, Suzuki Y, et al. (2010) Efficacy and safety of combination therapy of natural human interferon $B$ and ribavirin in chronic hepatitis $C$ patients with genotype 2 and high virus load. Inter Med 49: 965-970.

11. Nomura H, Miyagi $\mathrm{Y}$, Tanimoto $\mathrm{H}$, Yamashita $\mathrm{N}$, Oohashi $\mathrm{S}$, et al. (2012) Occurrence of clinical depression during combination therapy with pegylated interferon alpha or natural human interferon beta plus ribavirin. Hepatol Res 42: 241-247

12. Kumada H, Okanoue T, Onji M, Moriwaki H, Izumi N, et al. (2010) Guidelines for the treatment of chronic hepatitis and cirrhosis due to hepatitis $\mathrm{C}$ virus infection for the fiscal year 2008 in Japan. Hepatol Res 40: 8-13.

13. Ogawa E, Furusyo N, Toyoda K, Taniai H, Otaguro S, et al. (2010) Excellent superiority and specificity of COBAS TaqMan HCV assay in an early viral kinetic change during pegylated interferon alpha- $2 \mathrm{~b}$ plus ribavirin treatment BMC Gastroenterol 10: 38

14. [No authors listed] (1994) Intraobserver and interobserver variations in liver biopsy interpretation in patients with chronic hepatitis $\mathrm{C}$. The French METAVIR Cooperative Study Group. Hepatology 20: 15-20.

15. Ahmed WH, Furusyo N, Zaky S, Eldin AS, Aboalam H, et al. (2013) Pretreatment role of inosine triphosphate pyrophosphatase polymorphism for predicting anemia in Egyptian hepatitis C virus patients. World J Gastroenterol 19: 1387-1395.

16. Beck AT, Steer RA, Ball R, Ranieri W (1996) Comparison of Beck Depression Inventories -IA and -II in psychiatric outpatients. J Pers Assess 67: 588-597.

17. Buysse DJ, Reynolds CF 3rd, Monk TH, Berman SR, Kupfer DJ (1989) The Pittsburgh Sleep Quality Index: a new instrument for psychiatric practice and research. Psychiatry Res 28: 193-213.

18. Honda T, Katano Y, Shimizu J, Ishizu Y, Doizaki M, et al. (2010) Efficacy of peginterferon-alpha-2b plus ribavirin in patients aged 65 years and older with chronic hepatitis C. Liver Int 30: 527-537.

19. Evon DM, Ramcharran D, Belle SH, Terrault NA, Fontana RJ, et al. (2009) Prospective analysis of depression during peginterferon and ribavirin therapy of chronic hepatitis C: results of the Virahep-C study. Am J Gastroenterol 104 2949-2958.

20. Lang JP, Melin P, Ouzan D, Rotily M, Fontanges T, et al. (2010) Pegylated interferon-alpha2b plus ribavirin therapy in patients with hepatitis $C$ and psychiatric disorders: results of a cohort study. Antivir Ther 15: 599-606.

21. Furusyo N, Hayashi J, Ohmiya M, Sawayama Y, Kawakami Y, et al. (1999) Differences between interferon-alpha and -beta treatment for patients with chronic hepatitis C virus infection. Dig Dis Sci 44: 608-617.

22. Nicoletti F, Patti F, DiMarco R, Zaccone P, Nicoletti A, et al. (1996) Circulating serum levels of IL-1Ra in patients with relapsing remitting multiple sclerosis are normal during remission phases but significantly increased either during exacerbations or in response to IFN-beta treatments. Cytokine 8: 395-400.

23. Loftis JM, Huckans M, Ruimy S, Hinrichs DJ, Hauser P (2008) Depressive symptoms in patients with chronic hepatitis $\mathrm{C}$ are correlated with elevated plasma levels of interleukin-1beta and tumor necrosis factor-alpha. Neurosci Lett 430: 264-268.

24. Schloesser LL, Kipp MA, Wenzel FJ (1965) Thrombocytosis in iron-deficiency anemia. J Lab Clin Med 66: 107-114.

25. Akan H, Güven N, Aydogdu I, Arat M, Beksaç M, et al. (2000) Thrombopoietic cytokines in patients with iron deficiency anemia with or without thrombocytosis. Acta Haematol 103: 152-156.

26. Bilic E, Bilic E (2003) Amino acid sequence homology of thrombopoietin and erythropoietin may explain thrombocytosis in children with iron deficiency anemia. J Pediatr Hematol Oncol 25: 675-676. 